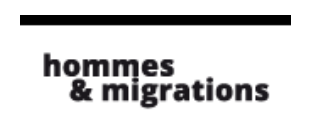

\section{Hommes \& migrations}

Revue française de référence sur les dynamiques

migratoires

$1304 \mid 2013$

Frontières

\title{
Faits divers, principe humanitaire et contrôle des migrants dans les Alpes du Sud (1850-1920)
}

\section{René Siestrunck}

\section{(apenEdition \\ Journals}

\section{Édition électronique}

URL : http://journals.openedition.org/hommesmigrations/2656

DOI : 10.4000/hommesmigrations.2656

ISSN : 2262-3353

\section{Éditeur}

Musée national de l'histoire de l'immigration

\section{Édition imprimée}

Date de publication : 1 octobre 2013

Pagination : 121-125

ISBN : 978-2-919040-24-7

ISSN : $1142-852 X$

\section{Référence électronique}

René Siestrunck, «Faits divers, principe humanitaire et contrôle des migrants dans les Alpes du Sud (1850-1920) », Hommes \& migrations [En ligne], 1304 | 2013, mis en ligne le 01 janvier 2017, consulté le 30 avril 2019. URL : http://journals.openedition.org/hommesmigrations/2656 ; DOI : 10.4000/ hommesmigrations.2656 


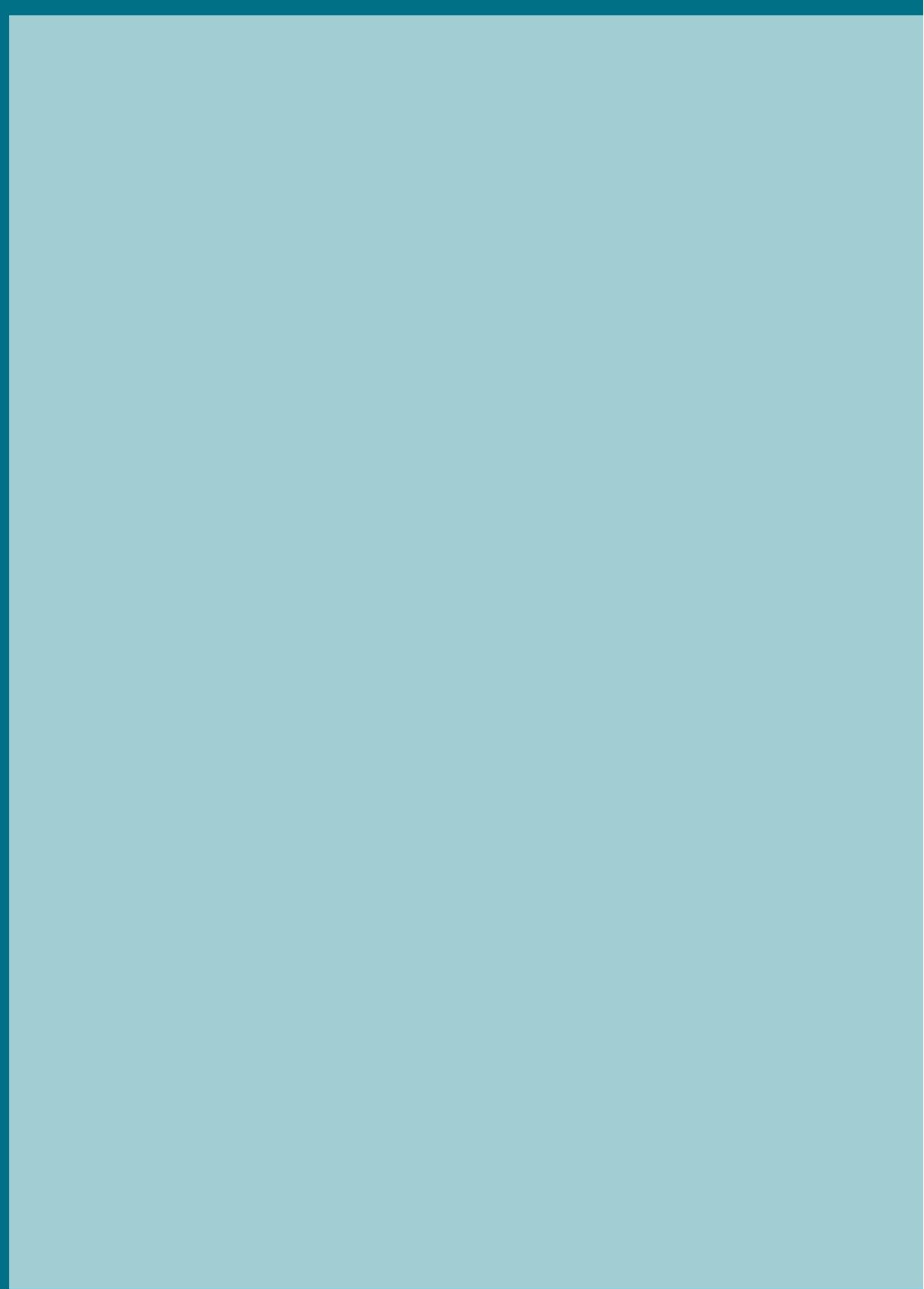

La Domenica del Corriere, 28 février 1932,

titre sur un drame fréquent : des migrants égarés

dans la tourmente

๑ René Siestrunck 


\title{
FAITS DIVERS, PRINCIPE HUMANITAIRE ET CONTRÔLE DES MIGRANTS DANS LES ALPES DU SUD (1850-1920)
}

par RENÉ SIESTRUNCK, MJC-Centre social du Briançonnais.

\author{
Au XIXe siècle, de nombreux Piémontais qui viennent chercher \\ du travail franchissent les cols des Alpes du Sud dans \\ les Hautes-Alpes. Face aux accidents fréquents en montagne, \\ refuges, forts et autres chapelles sont construits afin de \\ sécuriser les étapes de cette circulation migratoire. Les faits \\ divers font apparaître la réalité des déplacements humains \\ par-delà la frontière entre la France et l'Italie, tout à la fois \\ dangereuse et poreuse.
}

\section{Les drames de la montagne}

Longtemps ce genre de drame a relevé du fait divers. On approche du jour de Noël 1863. D’Abriès, dans le Queyras, un groupe de trois scieurs de long s'apprêtent à regagner leur village, Thures, commune de Bousson, en Italie, passant par le col de la Mayt, à 2706 mètres d'altitude ${ }^{1}$. Au cours des semaines précédentes, ils ont scié moult planches et poutres dans les villages des alentours. Ils ont en poche l'argent gagné depuis l'automne.

Partis à 4 heures, ils passent par le Roux d'Abriès et la Montette, un hameau inhabité. À Thures, ils sont attendus. Noël est une date importante, mais, s'il y a du travail, il faut le prendre. Alors on ne s'in- quiète pas tout de suite, mais tout de même. On écrit au maire d'Abriès qui répond qu'ils sont partis le 23 décembre. Depuis Thures et Abriès, on lance des recherches. Et trois semaines plus tard, on découvre, en bordure d'une avalanche, au-dessus du hameau de la Montette, des raquettes et un bâton.

Le juge de paix d'Aiguilles, Francou, est averti et prend toutes les mesures qui s'imposent, car ces "individus" portaient sur eux une certaine somme d'argent qui aurait pu donner des idées aux chercheurs de trésors. Il rend compte au procureur impérial, le 15 janvier 1864 : “Étant informé qu’ils 
devaient être porteurs de l'argent qu'ils avaient gagné en France, j'ai écrit à la Douane et à M. le maire d'Abriès pour faire exercer une surveillance active sur les lieux afin que rien ne soit détourné." Il explique aussi au procureur, en poste à Briançon, que les raquettes retrouvées non loin de l'avalanche sont des sortes de "chaussures dont on se L'inventaire macabre sert dans nos montagnes des accidents mortels permet d'établir la vraie géographie de l'occupation humaine de la montagne. pour pouvoir marcher sur la neige".

Le même jour, le juge avait dicté au maire d'Abriès la conduite à tenir : "Faites

faire toutes les recherches que vous jugerez convenables et que la saison peut comporter. Je pense que les habitants du Roux ne se refuseront pas à cette corvée. Dans le cas contraire, prenez procès-verbal contre les récalcitrants. Cependant, il ne faut pas que ces travaux les exposent à aucun danger." Et il prie le lieutenant des Douanes d'“inviter le brigadier du Roux à faire surveiller, autant que son service le permettra, les lieux où peuvent être les cadavres de ces trois individus".

Deux corps sont rapidement retrouvés, puis, plus tardivement, le troisième. Ils sont inhumés au Roux. Le 20 janvier 1864, le juge Francou rend compte au procureur impérial. L'identité et l'âge des victimes sont établis. Le premier a 64 ans, le second 37 et le troisième est encore plus jeune. "Ils sont malheureusement tous les trois pères de famille." Quant à leurs biens, "ils ont été remis à leurs parents".

\section{Le temps des refuges}

Ce fait divers dramatique n'est pas isolé et les pouvoirs publics s'en sont émus. C'est même une des vocations des refuges Napoléon, dont quatre d'entre eux sont construits en Queyras ou en lien avec lui (col Lacroix, col Agnel, col d'Izoard et col de Vars) ${ }^{2}$. Sur la route de Briançon à Grenoble, au col du Lautaret, remplaçant d'anciens abris existants depuis le Moyen Âge, un nouveau refuge est construit en 1863. La route est en construction et le refuge doit aussi abriter les ouvriers qui la construisent et les cantonniers qui l'entretiennent.

Lérudit briançonnais Aristide Albert, à la prose lyrique, s'émeut, en 1854, au spectacle des migrants qu'héberge l'ancien refuge, celui créé par les Dauphins au Moyen Âge, appelant à sa modernisation: "Le petit groupe défilait dans l'étroit sentier tracé dans la neige. En tête, le père chargé de tout le petit butin: quelques hardes, un peu de grain et de pommes de terre dans un sac. (...) Puis venait la pauvre femme, la mère, exténuée et triste à mourir. Elle portait sur son dos l'avant-dernier de ses enfants, pauvre petit ange dont les mains, attachées au cou de sa mère, étaient toutes rouges et gonflées de froid." En 1860, lors de son premier voyage dans les Alpes, le futur alpiniste Edward Whymper fait l'expérience du Lautaret et de son "misérable petit hospice", contrarié par la présence "d'ouvriers employés aux travaux de la route (...) dont les vêtements mouillés exhalaient les vapeurs les plus odieuses" (Escalades dans les Alpes, 1871.) Avec l'accroissement de la fréquentation touristique, on passe aussi de la promiscuité subie à un accueil spécialisé. Les équipements dédiés au voyageurs et migrants sont transformés pour les besoins de la nouvelle clientèle. Au cours d'une de ses dernières années d'activité d'alpiniste, William Auguste Coolidge, qui fréquente le massif et ses cols depuis vingt-cinq ans, dit en 1895 son étonnement devant les changements opérés dans l'hospice de 1863 : "Il y a maintenant vingt et une chambres, avec sonneries électriques, ainsi qu'un salon et une salle à manger assez grande, à laquelle on va encore construire une annexe $e^{3 .}$."

Au col Agnel, on en a le témoignage, le refuge a joué son rôle, même après avoir été transformé en poste militaire. "Lorsque souffle la terrible lombarde, malheur aux voyageurs!" écrit le lieutenant Mourrat ${ }^{4}$ qui commande le poste en 1895. Par 
beau temps, l'accueil des migrants italiens est commercial, avec tarifs affichés dans les deux langues. Mais, dans la tourmente, c'est au son du cor, voire à coups de fusil à blanc, que sont guidés les voyageurs égarés, à moins que tout l'effectif du poste ne se porte à leur secours. En décembre 1899, le gardien, seul au refuge, sauve deux Italiens parvenus à un kilomètre du refuge mais un de leurs compagnons meurt de froid.

L'inventaire macabre des accidents mortels permet d'établir la vraie géographie de l'occupation humaine de la montagne. On emprunte un col quelle que soit son altitude, quelle que soit la saison, parce qu'il est le plus court chemin entre deux villages ou parce qu'on l'a déjà franchi à la belle saison. Ainsi, cet ouvrier italien, parti durant l'hiver 1911 d'Abriès et se rendant dans son village de l'autre versant par le col de Valante (2815 mètres) et dont le corps est retrouvé au mois d'août suivant.

\section{Émergence de "la question humanitaire"}

Dans certaines circonstances, même les cols les plus fréquentés, les itinéraires "officiels" entre la France et l'Italie, peuvent se révéler dangereux. En novembre $1905^{5}$, trois Italiens qui tentaient de franchir le col de Montgenèvre ont dû leur salut à la caravane de secours composée d'habitants des Alberts, le village situé au pied du versant français. L'événement est assez important pour être signalé par La Montagne, revue officielle du Club alpin français (CAF). Les membres de cette institution puissante, à laquelle appartiennent de nombreux militaires, sont sensibles aux risques encourus par tous ceux qui fréquentent la montagne. L'occupation des postes d'hiver a donné l'occasion à certains d'entre eux d'assister, impuissants, à la répétition des drames. C'est le cas du lieutenant
Trémeau, en poste à la batterie de Viraysse, dans la Haute-Ubaye. Plusieurs cols relient le bassin de Cuneo et ses hautes vallées à la vallée de Barcelonnette. Le col de Sautron (2670 m) est le plus fréquenté et "vraiment homicide", selon les termes de l'appel lancé par le lieutenant Trémeau dans La Revue alpine, revue de la section lyonnaise du CAF (octobre 1901). Il décrit en détail le déplacement des migrants: "Au printemps, ce sont de longues caravanes d'hommes, de femmes et d'enfants qui viennent s'embaucher, pour la saison, sur le marché de Barcelonnette ${ }^{6}$." Les accidents surviennent au retour, effectué isolément, parfois en plein hiver.

Le lieutenant Trémeau a tenté de sensibiliser les communes, mais elles "opposent aux recherches une inertie calculée, craignant d'engager leurs secours". Les migrants ont aussi parfois entre eux "une indifférence qui dépasse les bornes de l'égoïsme”. Pour durcir son propos, il donne la liste de ces morts en montagne figurant aux registres de la paroisse de Chiappera, dans le val Maïra - 34 depuis 1792 -, auxquels s'ajoutent les migrants inconnus, disparus corps et biens, ou dont les corps ont été rapatriés dans d'autres localités.

Quelques mesures simples pourraient être prises : déclarer son départ pour le col en mairie ; donner comme mission officielle
Dans les montagnes, dans les hameaux d'alpage, les paysans rivalisent de piété et multiplient les chapelles. Certaines sont la conséquences d'un voeu de migrants égarés dans la tourmente. aux militaires des postes d'hiver de porter secours ; créer dans les villages un corps de brancardiers volontaires; construire un abri sous le col. C'est cette dernière suggestion que retient le Club alpin français. En septembre 1902, un refuge est inauguré qui "abritera, aux heures mauvaises, le transhumant lassé, lui redonnera force et courage, lui permettra de gagner le pays ami et de rejoindre, à l'automne, la maison paternelle, humble et toujours plus chère ${ }^{7 "}$. 


\section{La divine providence}

Dans les montagnes, dans les hameaux d'alpage, les paysans rivalisent de piété et multiplient les chapelles. Certaines sont la conséquences d'un vœu de migrants égarés dans la tourmente. Ainsi, la chapelle de Cotolivier, sur le versant italien du Briançonnais, entre Césane et Oulx, a été construite en 1899, sur les ruines d'une autre, après le vœu de voyageurs pris dans une tourmente dissipée par l'apparition de la Vierge.

À Fenils, autre village du versant italien, au pied du Montgenèvre, le "col officiel", mais également sur le chemin du col de Désertes qui jette directement dans la vallée de la Clarée en évitant Briançon, c'est Notre-Dame-de-Bon-Secours qui veille sur les passants. Elle a eu fort à faire au long des siècles, comme son homologue si-tuée dans la montée du col de l'Échelle, versant français, sous le vocable de Notre-Dame-de-Bon-Rencontre, même si la légende veut qu'elle ait été érigée par des marchands ayant échappé à des brigands. Reconnue comme la Sainte Vierge par les migrants pieux, l'apparition qui guide, dissipe les nuées et calme le vent peut être aussi l'une des nombreuses "dames blanches" peuplant sentiers et chemins et menant l'errant vers le salut ou à sa perte, selon leur humeur.

\section{Retours forcés}

Si les migrants se retrouvent sur les chemins à des dates convenues, selon leur activité, des événements peuvent contrarier cette régularité. C'est le cas des mouvements sociaux. On apprécie le travailleur piémontais. Le conducteur de travaux Potier, sur le chantier de la route du Lautaret, en fait l'éloge en 1847: "Les Piémontais sont de bons tailleurs de pierre, maçons, mineurs et carreyeurs, habitués à travailler dans le gneiss et le granit et on en dispose quand on en a besoin ${ }^{8}$." Les paysans du cru, eux aussi obligés d'émigrer en plaine jusqu'à fin mai, cultivent durant l'été et ne sont pas disponibles. En plus d'être "irréguliers, ils ne sont bons qu'à piocher la terre, charger et rouler des brouettes". Cette mauvaise volonté s'explique aussi par le fait que les entrepreneurs, pour emporter les marchés, rognent sur les frais de personnels et pour cette autre raison préfèrent les Piémontais, moins chers.

Ils sont tellement utiles, ces Piémontais, même devenus italiens, si adroits et doués pour toutes les sortes de maçonneries, qu'on les emploie même là où on les attend le moins : les fortifications. Peuton imaginer que les forts perchés sur les arêtes des Alpes sont bâtis par ceux-là mêmes qui, ayant revêtu l'uniforme de leur pays, devraient partir à leur conquête? L'effort de construction militaire est intense, surtout depuis l'adhésion de l'Italie à la Triplice, alliance avec l'Allemagne et l'Autriche signée en 1882. Sur les deux versants, les Alpes sont en chantier. Sur celui du fort de l'Olive, qui contrôle plusieurs cols avec l'Italie, les travailleurs italiens, qu'on continue à appeler "Piémontais", se mettent en grève. Le troupe monte alors de Briançon pour les ramener à la raison et les renvoie chez eux. Ils connaissent le chemin. À Briançon, en 1891, après diverses actions jugées vexatoires des douaniers italiens - on est en pleine guerre douanière franco-italienne -, la direction de l'usine de peignage de soie de la Schappe, qui emploie plus de 1000 personnes, accomplit un acte qu'elle considère comme hautement patriotique et "renvoie tous les ouvriers piémontais qui étaient employés dans ce vaste et important établissement industriel". La presse salue le geste et n'en attend pas moins de l'État: "C'est là un exemple que nos administrations devraient suivre, et surtout l'administration militaire. Espérons que l'on se décidera à chasser de nos chantiers ces étrangers, surveillants et ouvriers." "L'usine malgré tout fonctionnait grâce à une main-d'œuvre féminine, qu'on était allé chercher dans les vallées italiennes. Les soubresauts de la politique dérèglent les rythmes des migrations. 


\section{Vos papiers!}

Durant la guerre de 1914-1918, la France a fait largement appel à la main-d'œuvre des pays non belligérants. Des accords sont passés avec des pays neutres comme l'Espagne ou la Grèce. Cette population est très encadrée. Certificats et "passeports pour l'intérieur" délivrés par les mairies sont remplacés par un document unique, la carte d'identité, instaurée par deux décrets de 1917. Les Italiens, pendant la guerre, sont les alliés des Français. Sur le front italien, ils combattent côte à côte. À Briançon parviennent des convois de blessés soignés dans la cité. La guerre finie, il importe aux autorités de redonner consistance à la frontière, non par des obstacles matériels mais par des documents administratifs. Au cours de l'année 1920, dans les gares, dans les villages de pied de cols, les patrouilles de gendarmes redoublent leurs contrôles. Ils interpellent ces émigrés, établis pour certains depuis plus de dix ans en France, se sentant plus français qu'italiens, les ramenant à ce qu'ils sont : des sanspapiers. Ainsi, Bonifacio G., contrôlé à La Vachette, passage obligé à la descente du Montgenèvre, mineur à La Mûre, dans l'Isère, revenant de passer quelques jours de repos chez lui à Bussoleno, n'a ni passeport ni carte d'identité et n'a pas fait viser ses pièces avant son départ. Ils sont nombreux dans le même cas, à travailler régulièrement dans les mines et usines du Briançonnais (Villard-Saint-Pancrace, Saint-Crépin) ou en direction de Grenoble (Gavet, Livet, Vizille). "Je n'ai jamais eu de carte d'identité et j’ignorais que cette formalité était nécessaire pour résider en France", déclarent-ils en chœur sous la plume des gendarmes ${ }^{10}$. Le préfet des Hautes-Alpes avait anticipé sur ce contrôle en prenant, l'année précédente, un arrêté interdisant le passage du col de l'Échelle (l'un des plus bas des Alpes) à toutes personnes autres que celles munies d'une autorisation frontalière, tentant par là de réserver l'usage de ce passage aux particuliers, cultivateurs et éleveurs ayant des propriétés de part et d'autre, et d'orienter le flux des migrants vers le Montgenèvre, mieux surveillé. C'est le signe aussi que le mouvement migratoire du rural vers le rural se tarit, que cette émigration diffuse se concentre désormais vers les centres industriels. Aucun passant du col n'était informé d'avoir à faire viser ses papiers par le consul de France! Les destinations des interpellés du col de l'Échelle dessinent la géographie industrielle du quart sud-est de la France, des plus proches usines (L'Argentière et l'aluminium) aux fameuses usines du Rhône, passant par celles de Jarrie (chlore).

\section{Un accident décisif}

Un événement, qui n’a d’autre lien avec les migrations que le déplacement en montagne, a changé le statut du passage de col, audacieux ou mortel. Le 14 juillet 1865, le Cervin, jusqu'alors considéré comme inaccessible, était vaincu par une cordée de sept alpinistes. Mais, au cours de la descente, l'un d'entre eux glisse, en entraîne trois autres, Edward Whymper et deux guides ne devant leur salut quà la corde qui les reliait au groupe et qui cède. Avec le grand guide Michel Croz, les trois autres victimes sont anglaises, dont un jeune lord. L'émotion est vive en Angleterre. Dans l'entourage de la reine Victoria et dans la presse, on parle d'interdire l'alpinisme, cette pratique qui menace la fine fleur de l'empire. En Suisse, un juge enquête sur les circonstances du drame. L'accident de montagne change de statut. De simple fait divers, relaté avec fatalité comme on le ferait de l'accident d'un bûcheron écrasé par l'arbre qu'il abattait ou de la bergère foudroyée pendant un orage, il prend une autre signification. La porte est ouverte à la recherche d'une responsabilité, d'une faute, de la défaillance ou de l'absence d'un équipement. Et cette nouvelle manière de traiter de l'accident de montagne concerne alors tous les usagers de ce territoire, du dilettante fortuné au migrant qui fait sa trace sur le chemin du col quand souffle la lombarde. 